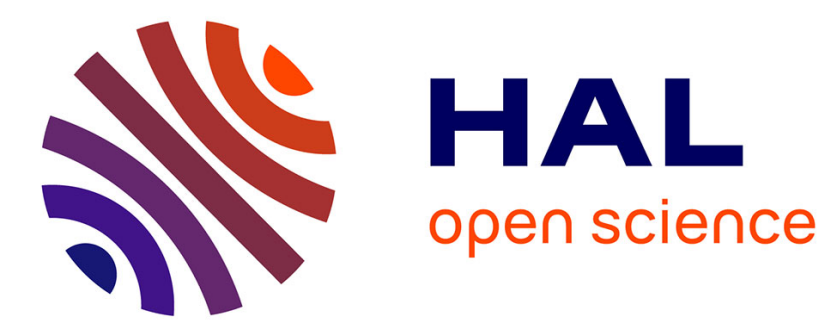

\title{
Design of Planar Resonant Scatterer With Roll-Invariant Cross Polarization
}

Olivier Rance, Nicolas Barbot, Etienne Perret

\section{To cite this version:}

Olivier Rance, Nicolas Barbot, Etienne Perret. Design of Planar Resonant Scatterer With RollInvariant Cross Polarization. IEEE Transactions on Microwave Theory and Techniques, 2020, 68 (10), pp.4305-4313. 10.1109/TMTT.2020.3014376 . hal-03235817

\section{HAL Id: hal-03235817 https://hal.science/hal-03235817}

Submitted on 26 May 2021

HAL is a multi-disciplinary open access archive for the deposit and dissemination of scientific research documents, whether they are published or not. The documents may come from teaching and research institutions in France or abroad, or from public or private research centers.
L'archive ouverte pluridisciplinaire HAL, est destinée au dépôt et à la diffusion de documents scientifiques de niveau recherche, publiés ou non, émanant des établissements d'enseignement et de recherche français ou étrangers, des laboratoires publics ou privés. 


\title{
Design of Planar Resonant Scatterer With Roll Invariant Cross Polarization
}

\author{
Olivier Rance, Nicolas Barbot, Member, IEEE, Etienne Perret, Senior Member, IEEE
}

\begin{abstract}
Only a few efforts have been made to design small scatterers with special polarimetric properties. In this article, the possibility of designing a planar single-layer resonant scatterer presenting roll invariant cross polarization is demonstrated for the first time. The invariance condition for a general scatterer is first derived analytically from the scattering matrix for co and cross polarization. Then a design method for a resonant scatterer made from two dipoles with unique angular and phase arrangement is presented. The first step of the design is done theoretically using field superposition. The effects of the coupling between the dipoles and the finite ground plane size are taken into account in a second step by simulation. Two scatterers operating at $4.26 \mathrm{GHz}$ and 5.26 GHz have been manufactured and measured providing good performance with a relative variation of their cross polarization component lower than $-2 \mathrm{~dB}$ and $-2.7 \mathrm{~dB}$ respectively. Finally, the scattering matrix corresponding to the current design is compared with other classical targets.
\end{abstract}

Index Terms - Cross polarization, Polarimetric Scattering, Resonator.

\section{INTRODUCTION}

$\mathrm{T}$ HE PRINCIPLE of polarimetric scattering has received significant attention in recent years in the field of remote electromagnetic sensors. Research has been driven by the rapid development of synthetic aperture radar imagery for radar target identification [1] and terrain classification [2]. In this field of interest, target decomposition schemes have been proposed with a special consideration for roll-invariant descriptors [3]-[5].

At the same time, advances in the fields of frequency selective surfaces and metamaterials have made it possible to produce engineered surfaces with specific polarimetric characteristics. Numerous applications have been reported in the literature, including polarimetric rotators [6], polarimetric converters [7] or metal-only diffractive surfaces [8], [9].

In contrast, little effort has been made to design small scattering particles with special polarimetric properties. In this article, a design method for a planar resonant target whose signature is invariant by rotation along the line of sight (roll invariant) is proposed. Although the design of planar roll invariant scatterer in co polarization (symmetric scattering) is fairly straightforward, it is more challenging to design roll invariant planar scatterers in cross polarization (asymmetric

This work is funded by the European Research Council (ERC) under the European Union's Horizon 2020 research and innovation program (Grant No 772539). scattering) and, at the authors' knowledge, this type of target has never been reported in the literature.

The single layered scatterer proposed in the article is resonant and finds a direct application in the field of Chipless RFID. In Chipless RFID, small resonant scatterers are used to encode information. The necessity to isolate the field backscattered by the scatterer from that of the environment has led to new reading techniques exploiting cross polarization. For instance, in [10] a depolarizing scatterer is used in combination with a cross polarization measurement to provide a robust reading, even for a label attached to a metallic object. At present, the orientation of the scatterer has to be known in advance and the use of cross polarization roll invariant scatterers would allow for more flexible reading in this context.

In the article, the roll invariant condition is theoretically established for a completely general scatterer. The approach could therefore potentially be transposed to other application areas such as polarimetric radar calibration or radar cross section reduction (RCSR) techniques.

Currently, the calibration of a polarimetric radar is performed using reference targets such as the dihedral corner or the wire mesh [11], [12] which must be precisely aligned at an angle of $22.5^{\circ}$ and $45^{\circ}$ to the antennas, respectively, in order to produce the desired cross polarization component. A roll invariant scatterer may be beneficial as a reference object for calibration to alleviate the constraint of accurate positioning.

In the domain of RCSR, the wave backscattered by a conventional radar absorbing material such as [13] generally has the same polarization as the incoming wave. Being able to evenly distribute the backscattered power among co and cross components would result in a direct reduction of the reflection coefficient by $3 \mathrm{~dB}$. In addition, new types of metallic-only diffractive surfaces have been reported in the literature [8], [9], which avoid the use of expensive resistive layers. These structures are based on the juxtaposition of different sectors producing a phase difference of $180^{\circ}$ at broadside. A way to achieve more diffuse scattering is to break the periodicity and, for some examples [8], [9], to break the symmetry of the initial checkerboard. Although promising, some of these structures are currently orientation-sensitive in that they suffer performance degradation when the incoming wave is not polarized in a specific direction. The method proposed in this article could be of interest for designing new types of frequency selective surfaces with roll invariant unit cells to solve the problem of orientation sensitivity.

The authors are with Univ. Grenoble Alpes, Grenoble INP, LCIS, F-26000 Valence, France. M. Perret is also with Institut Universitaire de France, Paris, France. 
In the article, we first analytically determine the general condition allowing rotational invariance of the cross polarization component from the examination of the polarimetric scattering matrix for a scatterer under normal incidence. Next, a simple design method based on the use of two microstrip dipoles is proposed for the realization of a rollinvariant resonant scatterer. The scatterer is finally fabricated and the performance obtained is evaluated from measurements.

\section{THEORY}

The interaction between the incident wave and the radar target can be described by the polarization scattering matrix $\boldsymbol{S}$ (Sinclair matrix) which relates the scattered electric field vector $\boldsymbol{E}^{s}$ to the incident field vector $\boldsymbol{E}^{i}$ :

$$
\left[\begin{array}{l}
E_{v}^{S} \\
E_{h}^{S}
\end{array}\right]=\frac{e^{-j k r}}{\sqrt{4 \pi} \cdot r} \cdot\left[\begin{array}{ll}
S_{v v} & S_{v h} \\
S_{h v} & S_{h h}
\end{array}\right] \cdot\left[\begin{array}{c}
E_{v}^{i} \\
E_{h}^{i}
\end{array}\right],
$$

where $S_{v v}, S_{h h}, S_{v h}$ and $S_{h v}$ are complex frequency-dependent quantities. $r$ is the distance between the target and the antenna and $k$ is the wave number. For a passive target in monostatic configuration (see Fig. 1), $\boldsymbol{S}$ is symmetric i.e. $S_{v h}=S_{h v}$. In (1) the range dependence $r$ is factored out of the $S$ matrix to be consistent with the classical definition of the polarimetric radar cross section.

When the target is rotated by an angle $\theta$ along the line of sight (see Fig. 1), the scattering matrix is modified according to:

$$
\boldsymbol{S}(\theta)=\boldsymbol{\Omega}^{t} \cdot \boldsymbol{S}(0) \cdot \boldsymbol{\Omega},
$$

where $\boldsymbol{\Omega}^{t}$ denotes the transpose of $\boldsymbol{\Omega}$ which is the rotation matrix of angle $\theta$ :

$$
\boldsymbol{\Omega}=\left[\begin{array}{cc}
\cos \theta & -\sin \theta \\
\sin \theta & \cos \theta
\end{array}\right]
$$

For the rest, it will prove useful to derive from (2) the explicit form of $S_{v v}$ (co polarization) and $S_{v h}$ (cross polarization) with respect to $\theta$ :

$$
\begin{aligned}
S_{v v}(\theta)= & \frac{S_{h h}+S_{v v}}{2} \\
& -\frac{S_{h h}-S_{v v}}{2} \cos 2 \theta+S_{v h} \sin 2 \theta, \\
S_{v h}(\theta) & =\frac{S_{h h}-S_{v v}}{2} \sin 2 \theta+S_{v h} \cos 2 \theta .
\end{aligned}
$$

In (4) and (5), the values $S_{v v}, S_{h h}$, and $S_{v h}$ are the components of the scattering matrix $S$ for the reference orientation of the target $\theta=0$. For a general scatterer, the components of the scattering matrix depend on the roll angle $\theta$. In the following two sections, we seek to establish the conditions of invariance for co and cross polarization.

\section{A. Co polarization component invariant by rotation}

In this section, we are looking for the conditions on the components of the scattering matrix such that $S_{v v}$ remains constant by rotation, which mathematically translates into:

$$
\exists a \in \mathbb{C}, \quad \forall \theta, \quad S_{v v}(\theta)=a .
$$

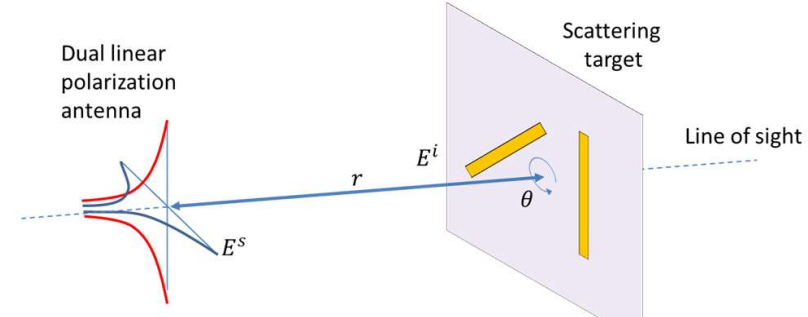

Fig. 1. Radar target under normal incidence. Monostatic configuration.

Applying the invariance condition (6) for three specific angles $\theta=0, \theta=\pi / 2$, and $\theta=\pi / 4$ in (4) gives the system:

$$
\left\{\begin{array}{l}
S_{v v}=a \\
S_{h h}=a \\
S_{v h}=0
\end{array}\right.
$$

From which it is clear that the $S$ matrix takes the form:

$$
\boldsymbol{S}(0)=a \cdot \boldsymbol{I},
$$

where $I$ is the $2 \times 2$ identity matrix. In this case $S$ commutes with $\boldsymbol{\Omega}$ in (2) so that (8) remains valid for all angle values $\theta$.

Planar geometries having this property are either non resonant targets for which the RCS is proportional to the metallization surface or resonators presenting $n$-folded rotational symmetries [14]. A 3D target also sharing this property is the conducting sphere, which is typically used as a reference element for polarimetric radar calibration.

\section{B. Cross polarization component invariant by rotation}

In cross polarization, the search for invariance for both magnitude and phase leads straightforwardly to the condition $S_{v h}=0$ for all $\theta$. More interesting results are obtained for a weaker formulation of the problem which considers only the invariance of the magnitude:

$$
\exists b \in \mathbb{R}^{+}, \quad \forall \theta, \quad\left|S_{v h}(\theta)\right|=b .
$$

Applying the invariance condition (9) for three specific angles $\theta=0, \theta=\pi / 4$, and $\theta=\pi / 8$ in (5) reduces to the system:

$$
\left\{\begin{array}{l}
\left|S_{v h}\right|=b \\
\left|\frac{S_{h h}-S_{v v}}{2}\right|=b \\
\frac{S_{h h}-S_{v v}}{2} \cdot S_{v h}=0
\end{array}\right.
$$

From (10), we see that the $\theta$-independent weight terms in (5) must be of same magnitude and have a phase offset of $\pi / 2$, which can be written in a more compact form:

$$
\frac{S_{h h}-S_{v v}}{2}= \pm j \cdot S_{v h}
$$

If we replace $\left(S_{h h}-S_{v v}\right) / 2$ by $\pm j \cdot S_{v h}$ in (5), we see that $S_{v h}(\theta)$ is describing an origin centered circle in the complex plane, which ensures the magnitude invariance for any $\theta$. The sign chosen in (11) determines the direction of rotation. It is interesting to note that, for this condition, the scattering matrix 
can be decomposed as:

$$
\boldsymbol{S}=\frac{S_{h h}+S_{v v}}{2} \cdot \boldsymbol{I}+\frac{S_{h h}-S_{v v}}{2} \cdot \boldsymbol{S}_{h},
$$

where the first term of the summation corresponds to the copolarization roll-invariant case (with no component in cross polarization) described in the previous section and the second term of the summation has the exact same form as the scattering matrix of a 3D helix:

$$
\boldsymbol{S}_{h}=\left[\begin{array}{cc}
1 & \pm \mathrm{j} \\
\pm \mathrm{j} & -1
\end{array}\right]
$$

These two matrixes have been classically used as basis decomposition elements for radar target identification [15].

\section{DESIGN}

The design of a radar target corresponding to a given polarimetric scattering matrix is generally a difficult inverse problem. Here, a simple method is proposed to design a target with a cross polarization insensitive to rotation using two microstrip dipoles.

\section{A. Principle}

We start by noticing that applying a rotation angle $\theta=\pi / 4$ in (5) gives:

$$
S_{v h}(\pi / 4)=\frac{S_{h h}-S_{v v}}{2}
$$

Such that (5) can be rewritten:

$$
S_{v h}(\theta)=S_{v h}(\pi / 4) \cdot \sin 2 \theta+S_{v h}(0) \cdot \cos 2 \theta .
$$

Equation (15) gives a simple geometric interpretation to the cross polarization invariance condition (11): the cross components of the scatterer oriented at 0 and $\pi / 4$ have to be orthogonal with the same magnitude. This formulation has the advantage of not mixing co and cross terms in the same equation as was done in (5).

A natural idea is then to consider a target composed of two dipoles respectively oriented at 0 and $\pi / 4$ as represented schematically in Fig. 2. In this configuration, if the couplings between the two adjacent dipoles are neglected, only the first dipole has a cross contribution when the target is oriented at 0 (Fig. 2a) and only the second dipole has a cross contribution when the target is oriented at $\pi / 4$ (Fig. 2b). Note also that the magnitude and phase of $S_{v h}$ can thus be set independently for the two orientations of interest by adjusting the width and length of dipole $n^{\circ} 1$ and $n^{\circ} 2$ respectively.

The phase quadrature between the two dipoles is obtained by slightly shifting their resonant frequencies, as shown in Fig. 3. In this manner the phase of the two resonators appears to be respectively advanced or delayed by about $\pi / 4$ at the operating frequency $f_{0}$. The frequency shift $\Delta f$ and the corresponding incremental length $\Delta L$ required to achieve a phase offset of $\pi / 2$ can be calculated simply by modeling the resonance with the canonical expression of a second-order resonator:

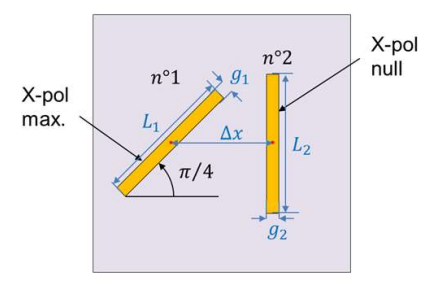

(a)

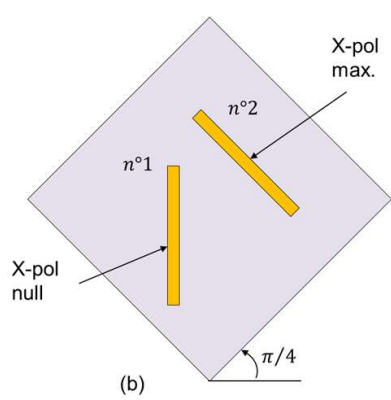

(b)
Fig. 2. Cross-polarization contribution of a target composed of two microstrip dipoles oriented at 0 (a) and $\pi / 4$ (b). (b)

Fig. 3. Phase quadrature between two microstrip resonators having shifted resonant frequencies. The corresponding length variation depends on the quality factor $Q$ of the resonators.

$$
S_{v h}(f)=\frac{A}{1+j Q\left(\frac{f}{f_{0}}-\frac{f_{0}}{f}\right)}
$$

and using a first order approximation of the phase around $f_{0}$ (see Fig. 3b), which gives:

$$
\frac{\Delta f}{f_{0}}=\frac{\Delta L}{L}=\frac{\pi}{8 \cdot Q} .
$$


When the two resonators are shifted symmetrically $\left(f_{1,2}=f_{0} \pm\right.$ $\Delta f$ ) as in Fig. 3a, they happened to have the same magnitude $A^{\prime}$ at $f_{0}$ which can be calculated from (16) and (17) as:

$$
A^{\prime}=A \cdot\left[1+\left(2 Q \frac{\Delta f}{f_{0}}\right)^{2}\right]^{-\frac{1}{2}}=\frac{A}{\sqrt{1+\pi^{2} / 16}}
$$

Equation (18) shows that the approach gives a magnitude decay of $A^{\prime}$ compared to $A$ of approximately $-2 \mathrm{~dB}$ which does not depend on either the operating frequency $f_{0}$ or the quality factor $Q$ of the resonator.

When the two dipoles are arranged as described in Fig. 2a with the proper phase offset (with resonator $n^{\circ} 2$ being the phase reference), their scattering matrixes at $f_{0}$ are given respectively by:

$$
\begin{aligned}
& \boldsymbol{S}_{1}=-j A^{\prime} \cdot\left[\begin{array}{ll}
1 & 1 \\
1 & 1
\end{array}\right] \\
& \boldsymbol{S}_{2}=2 A^{\prime} \cdot\left[\begin{array}{ll}
1 & 0 \\
0 & 0
\end{array}\right] .
\end{aligned}
$$

If we neglect the couplings, the scattering matrix of the complete target is given by $\boldsymbol{S}_{1}+\boldsymbol{S}_{2}$ from superposition principle. This can be written as:

$$
\boldsymbol{S}=A^{\prime}(1-j) \cdot \boldsymbol{I}+A^{\prime} \cdot \boldsymbol{S}_{h} .
$$

Equation (20) has the same form as (12), which tends to confirm the design approach.

\section{B. Simulation results}

The scatterer consists of two microstrip dipoles printed on RO4003 substrate $\left(\varepsilon_{r}=3.55, \tan \delta=2.7 \cdot 10^{-3}\right)$ of thickness $0.8 \mathrm{~mm}$. The width $g_{1,2}$ of the dipoles (Fig. $2 \mathrm{a}$ ) is fixed at $2 \mathrm{~mm}$ and their length is chosen so that the corresponding resonant frequency is approximately $3.19 \mathrm{GHz}\left(L_{1,2} \simeq 26.9 \mathrm{~mm}\right)$. The separation $\Delta x$ between the centers of the dipoles is $20 \mathrm{~mm}$. For these dimensions, the quality factor of the individual (uncoupled) dipoles is evaluated to $Q=195$ from the classical cavity model [16]. The value of $\Delta L$ required for a phase offset of $90^{\circ}$ calculated from (17) is approximately $50 \mu \mathrm{m}$.

A series of simulations (Time domain solver of CST Microwave Studio 2018) has been carried out to dimension the two dipoles in the presence of coupling. The length of the dipole $\mathrm{n}^{\circ} 1$ is fixed at $L_{1}=26.9 \mathrm{~mm}$ and the length $L_{2}$ of the dipole

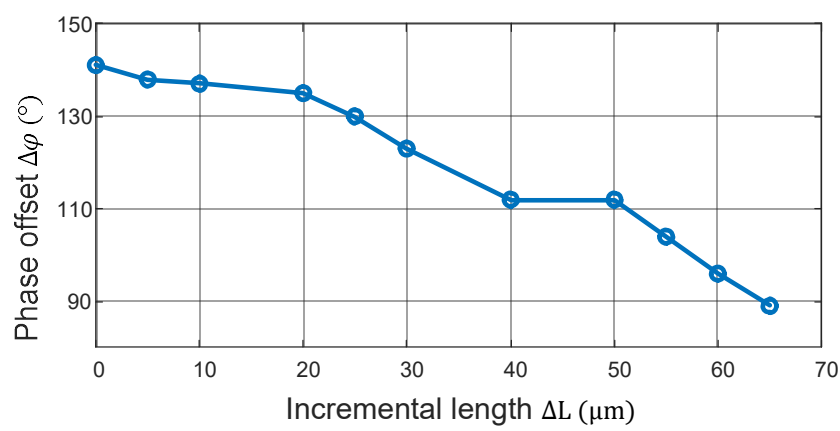

Fig. 4. Phase offset between $S_{v h}\left(0^{\circ}\right)$ and $S_{v h}\left(45^{\circ}\right)$ as a function of the incremental length. $L_{1}=26.9$ and $L_{2}=L_{1}+2 \Delta L, f_{0}=3.19 \mathrm{GHz}$ $\mathrm{n}^{\circ} 2$ is increased iteratively in steps of $10 \mu \mathrm{m}$ (note that $L_{2}-$ $\left.L_{1}=2 \Delta L\right)$. For each value of $\Delta L$, the backscattered field in cross polarization is computed for the angles $\theta=0$ and $\theta=$ $45^{\circ}$. The phase offset $\Delta \varphi$ at $f_{0}$ is reported in Fig. 4 as a function of the incremental length $\Delta L$. The phase offset of $90^{\circ}$ is obtained at $3.19 \mathrm{GHz}$ for $L_{1}=26.9 \mathrm{~mm}$ and $L_{2}=27.03 \mathrm{~mm}(\Delta L=65$ $\mu \mathrm{m})$.

For these dimensions, the $S_{v h}$ component of the scattering matrix has been computed for different orientations covering the range $\left[0^{\circ}, 180^{\circ}\right]$. The simulation results are represented in Fig. 5. As expected from the theory, all curves are almost concurrent at the operating frequency $f_{0}=3.19 \mathrm{GHz}$. Simulations show that the ground plane has a significant influence on the magnitude and frequency of the peaks when its lateral dimension is smaller than $2 \cdot \lambda_{0}$. For the current design, the ground plane has a lateral dimension of $50 \mathrm{~mm}\left(0.53 \cdot \lambda_{0}\right)$ and is responsible for the asymmetry of the peak magnitude observed between $\theta=0^{\circ}$ and $\theta=45^{\circ}$ in Fig. 5 .

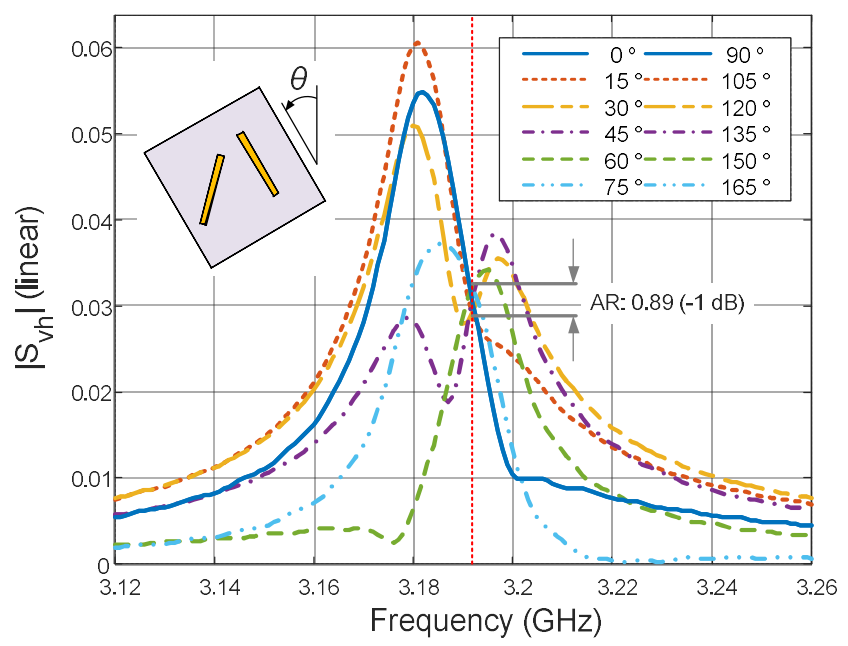

Fig. 5. Cross-polarization component of the scattering matrix with respect to frequency for different values of $\theta$ (CST simulation). $L_{1}=26.9 \mathrm{~mm}$ and $L_{2}=$ $27.03 \mathrm{~mm}$.

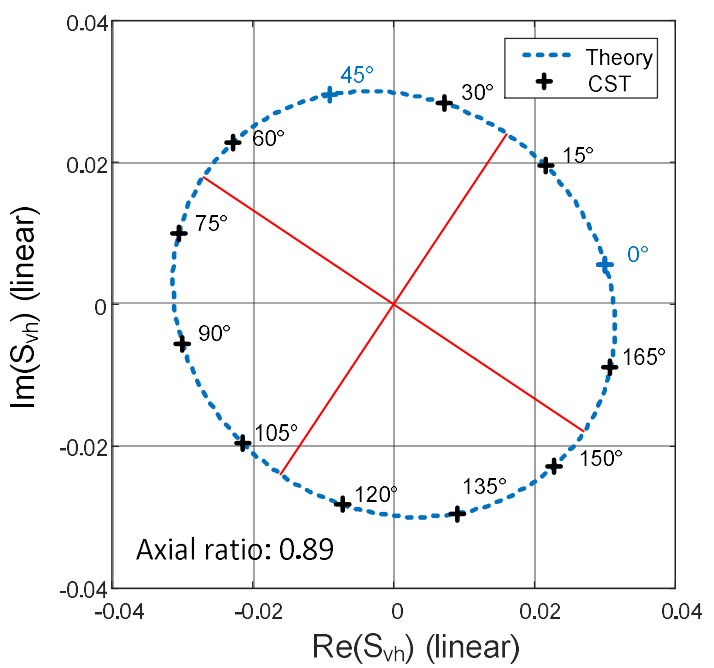

Fig. 6. Evolution of $\mathrm{S}_{\mathrm{vh}}$ as a function of the orientation angle $\theta$ in the complex plane. The theory is calculated from (15) and the computed values of $S_{v h}$ at $\theta=0^{\circ}$ and $\theta=45^{\circ}$. The CST simulation results correspond to the data presented in Fig. 4 evaluated at $\mathrm{f}_{0}=3.19 \mathrm{GHz}$ 
The trajectory of $S_{v h}(\theta)$ in the complex plane is calculated theoretically from (15) and the computed value of $S_{v h}$ at $\theta=$ $0^{\circ}$ and $\theta=45^{\circ}$ in Fig. 6. The simulated data (magnitude and phase at $f_{0}$ ) are also reported in Fig. 6 . We observe a very good agreement between theory and simulations. The axial ratio of the ellipse (minor axis on major axis, which are represented by red lines in Fig. 6) obtained by simulation for these values is $A R=0.89(-1 \mathrm{~dB})$. Note that a simple way to evaluate the axial ratio without plotting the ellipse is to calculate the ratio between the minimum and maximum magnitude at the operating frequency as indicated in Fig. 5.

\section{Sensitivity}

Fig. 4 shows that the phase offset $\Delta \varphi$ is very sensitive to the length of the dipoles. In the vicinity of $\Delta \varphi=90^{\circ}$, a step of only $10 \mu \mathrm{m}$ introduces an absolute phase error $\psi=16^{\circ}(18 \%$ relative error). A precision of $10 \mu \mathrm{m}$ is generally inferior to the manufacturing tolerances for classical PCB fabrication process. The design may also be subject to discrepancy if the two curves corresponding to $\theta=0^{\circ}$ and $\theta=45^{\circ}$ are not intersecting perfectly at the operating frequency (i.e. if there is a magnitude difference $A_{1}^{\prime} \neq A_{2}^{\prime}$ at $f_{0}$ ). In these cases, the trajectory of $S_{v h}(\theta)$ does not describe a perfect circle but an ellipse in the complex plane. From (15), it is possible to derive analytically the evolution of the minor axis and major axis values $\left(A_{m}\right.$ and $B_{m}$ ) of the ellipse with respect to $A_{1}^{\prime}, A_{2}^{\prime}$ and $\psi$ :

$$
\begin{aligned}
& A_{m}=\frac{A_{1}^{\prime}+A_{2}^{\prime}}{\sqrt{2}} \cos \left(\frac{\pi}{4}-\frac{\psi}{2}\right)+\frac{A_{1}^{\prime}-A_{2}^{\prime}}{\sqrt{2}} \cos \left(\frac{\pi}{4}+\frac{\psi}{2}\right) \\
& B_{m}=\frac{A_{2}^{\prime}-A_{1}^{\prime}}{\sqrt{2}} \cos \left(\frac{\pi}{4}-\frac{\psi}{2}\right)+\frac{A_{1}^{\prime}+A_{2}^{\prime}}{\sqrt{2}} \cos \left(\frac{\pi}{4}+\frac{\psi}{2}\right)
\end{aligned}
$$

Equations (21) and (22) thus make it possible to visualize the evolution of the axial ratio in the presence of errors either on the magnitude or on the phase as shown in Fig. 7.

We see that the axial ratio decreases almost linearly with respect to the phase error $\psi$ when $A_{1}^{\prime}=A_{2}^{\prime}$. Given the sensitivity of $\Delta \varphi$ to the length of the dipoles, achieving an axial

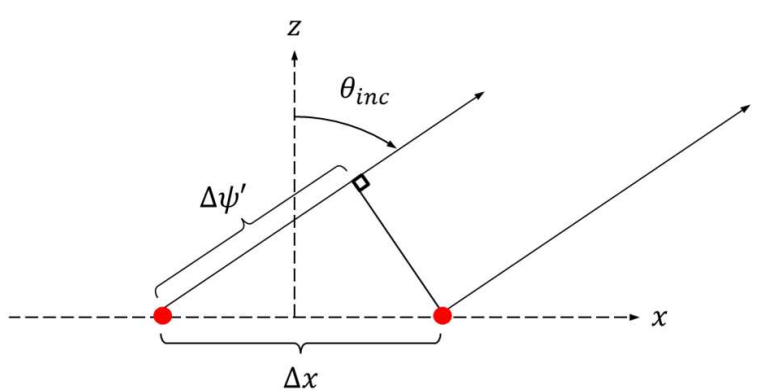

Fig. 8. Transverse view of the planar scatterer under oblique incidence for the derivation of (23)

ratio superior to $-3 \mathrm{~dB}$ seems to be a realistic objective for realization. Note that the value of $3 \mathrm{~dB}$ is a classical specification for circularly polarized antennas.

It is also interesting to evaluate the degradation of the axial ratio when the scatterer is under oblique incidence. The angle of incidence from broadside is denoted $\theta_{i n c}$ and the separation between the dipole centers is $\Delta x$ (see Fig. 8). At oblique incidence the spatial phase delay $\Delta \psi^{\prime}$ formed between the two dipoles disturbs the required $90^{\circ}$ phase offset:

$$
\Delta \psi^{\prime}=k_{0} \cdot \Delta x \cdot \sin \theta_{\text {inc }} .
$$

where $k_{0}$ is the wave number at the operating frequency. If we set an objective of $-3 \mathrm{~dB}$, it is seen from Fig. 7 that the phase delay has to be $\Delta \psi^{\prime} \leq 0.5 \cdot 20^{\circ}$ (the factor 0.5 is due to the return trip). For $f_{0}=3.19 \mathrm{GHz}$ and $\Delta x=20 \mathrm{~mm}$, the inversion of (23) gives an angular range validity of $\left[-7.5^{\circ}, 7.5^{\circ}\right]$.

\section{MEASUREMENT RESUlts}

\section{A. Measurement Setup}

Two scatterers (see Fig. 9) operating at $4.26 \mathrm{GHz}$ and $5.26 \mathrm{GHz}$ respectively have been manufactured by chemical etching. The substrate is a square board of RO4003 material with a width of $0.8 \mathrm{~mm}$ and lateral dimensions of $50 \mathrm{~mm} \times 50 \mathrm{~mm}$. The geometrical dimensions of the dipoles are given in Table I.

TABLE I

GEOMETRICAL DIMENSIONS AND MEASURED CHARACTERISTICS OF THE MANUFACTURED SCATTERERS

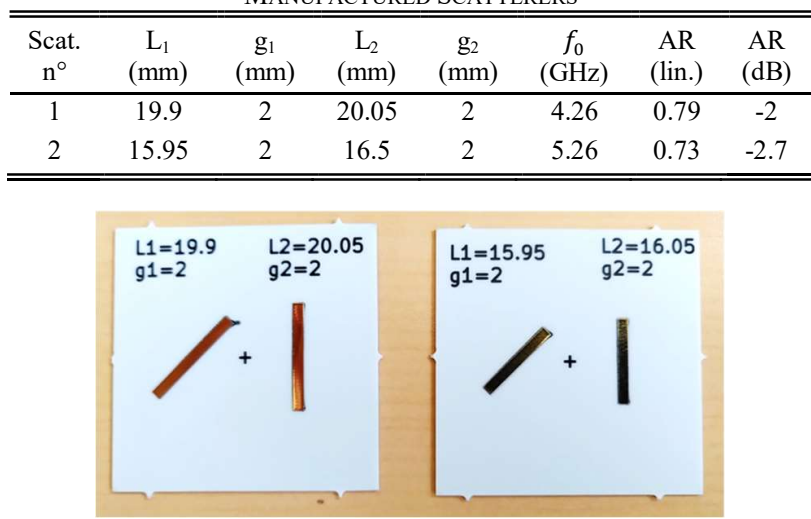

Fig. 9. Manufactured scatterers. Substrate is a square board of RO4003C dielectric of thickness $h=0.8 \mathrm{~mm}$ and lateral dimensions $50 \mathrm{~mm} \times 50 \mathrm{~mm}$ backed with a ground plane.
Fig. 7. Evolution of the axial ratio with respect to phase and magnitude errors. 


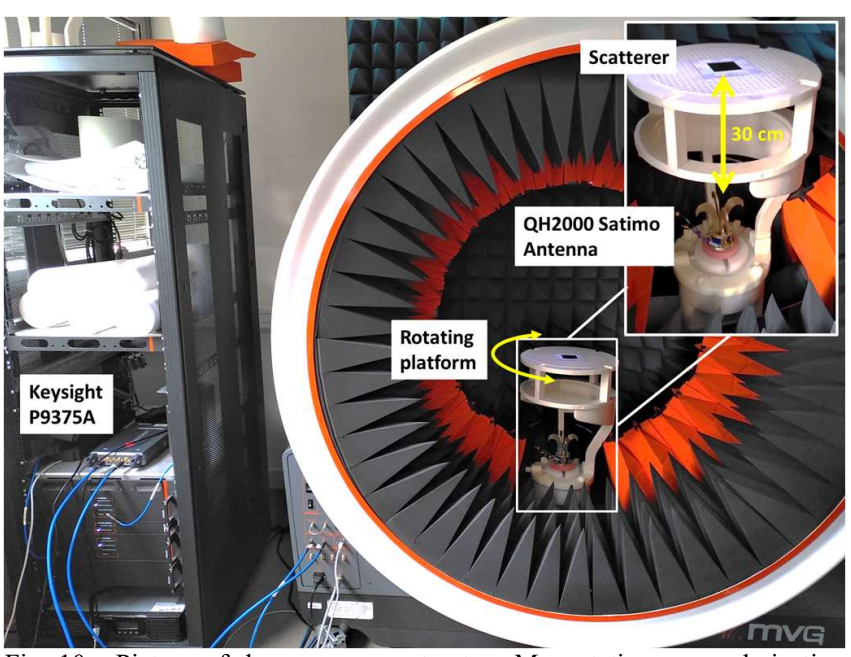

Fig. 10. Picture of the measurement setup. Monostatic cross polarization measurement in semi anechoic environment with the scatterer positioned on a rotating platform $30 \mathrm{~cm}$ away from the antenna.

The measurements are carried out in a semi anechoic environment using a modified MVG Starlab system, as represented in Fig. 10. The setup is monostatic and the measurements are done in cross polarization using a QH2000 quadridge horn antenna. The cross polarization isolation of the dual polarization antenna is $-35 \mathrm{~dB}$ and $-32 \mathrm{~dB}$ at $4.26 \mathrm{GHz}$ and $5.26 \mathrm{GHz}$ respectively. The scatterer under test is positioned on a rotating platform $30 \mathrm{~cm}$ away from the antenna. The Fraunhofer region for this antenna is reached for $r=31 \mathrm{~cm}$ and $r=38 \mathrm{~cm}$ at $4.26 \mathrm{GHz}$ and $5.26 \mathrm{GHz}$ respectively so that both scatterers are in the radiative near field of the antenna. In this region, the radiation pattern of the antenna is not well defined and depends on the reading distance. Compared to the far field radiation pattern, the largest inaccuracies arise in direction of nulls or low sidelobes, but a small influence is expected for the main beam [17]. It should be noticed that due to the small size of the scatterer $(5 \mathrm{~cm} \times 5 \mathrm{~cm})$, the antenna is located in the far field of the scatterer $(r=14 \mathrm{~cm}$ and $r=17.5 \mathrm{~cm}$ for 4.26 GHz and 5.26 GHz respectively), so that the backscattered wave can be considered transverse with correctly defined polarization.

The antenna is connected to a Keysight P9375A VNA which is interfaced to a laptop.

Although theoretically zero, the cross-polarization component of the wave backscattered by the ground plane is comparable to the one of the dipoles in measurement. In order to remove the ground plane contribution, a reference measurement containing only the ground plane (no dipoles) is performed for each angle and a complex subtraction is operated with the scatterer measurement. For many applications, the reference measurement of a ground plane having the same size and position as the scatterer is unpractical. In this case, other separation techniques may be more appropriate [18].

The measured $S_{21}$ parameter of the VNA is related to the $S_{v h}$ component of the scattering matrix by the monostatic radar equation:

$$
S_{21}=\frac{G \cdot \lambda}{(\sqrt{4 \pi})^{3} \cdot r^{2}} S_{v h},
$$

where $G$ is the antenna gain $(5.8 \mathrm{dBi}$ and $8.2 \mathrm{dBi}$ at $4.26 \mathrm{GHz}$ and $5.26 \mathrm{GHz}$ respectively) and $\lambda$ the wavelength in free space.

\section{B. Measurement Results}

Measurements of both scatterers have been performed for $\theta$ varying from $0^{\circ}$ to $180^{\circ}$ with steps of $5^{\circ}$. The measurement results are represented in Fig. 11a and Fig. $11 \mathrm{~b}$ for scatterers $\mathrm{n}^{\circ} 1$ and $\mathrm{n}^{\circ} 2$ respectively. A minimum variation of the magnitude is obtained at $4.26 \mathrm{GHz}$ (respectively $5.26 \mathrm{GHz}$ for scatterer $\mathrm{n}^{\circ} 2$ ) for which $\left|S_{v h}\right|$ is varying between $17.3 \cdot 10^{-3}$ and $13.7 \cdot 10^{-3}$ which corresponds to an axial ratio of $-2 \mathrm{~dB}$ $\left(19.2 \cdot 10^{-3}\right.$ and $14.1 \cdot 10^{-3}$ corresponding to $-2.7 \mathrm{~dB}$ for scatterer $n^{\circ} 2$ ). The magnitude decay between the peak apex value at $4.24 \mathrm{GHz}$ (resp. $5.24 \mathrm{GHz}$ for res $n^{\circ} 2$ ) and the average value at $f_{0}$ is approximately $-4.6 \mathrm{~dB}$ (resp. $-3 \mathrm{~dB}$ for scatterer $\mathrm{n}^{\circ} 2$ ) which is more than the $-2 \mathrm{~dB}$ expected in theory (18) from the model without coupling.

(a)

(b)

Fig. 11. Measurement results of the cross polarization component of the scattering matrix with respect to frequency for different values of $\theta$. (a) Scatterer $\mathrm{n}^{\circ} 1$ having an operating frequency $f_{0}=4.26 \mathrm{GHz}$. (b) Scatterer $\mathrm{n}^{\circ} 2$ having an operating frequency $f_{0}=5.26 \mathrm{GHz}$. 


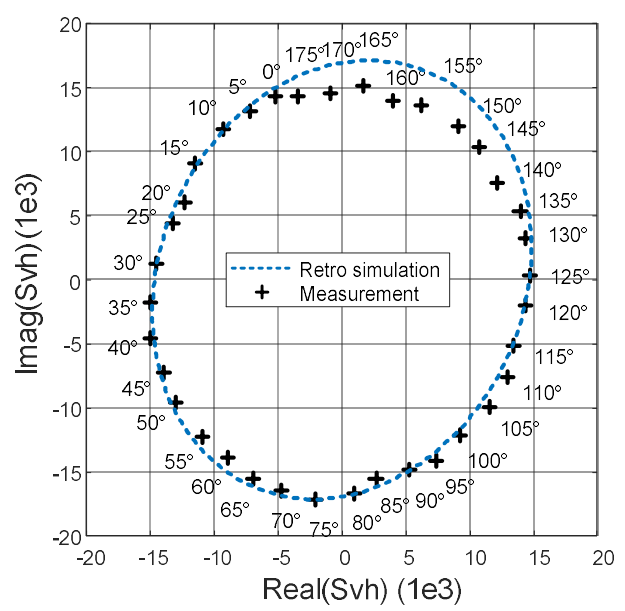

(a)

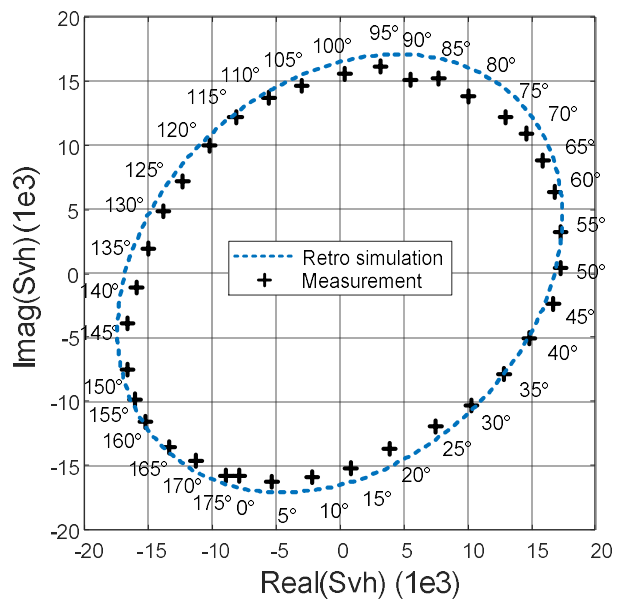

(b)

Fig. 12. Evolution of $\mathrm{S}_{\mathrm{vh}}$ at the operating frequency as a function of the orientation angle $\theta$ in the complex plane. (a) Scatterer $n^{\circ} 1, f_{0}=4.26 \mathrm{GHz}$, $\mathrm{AR}=0.79(-2 \mathrm{~dB})$. Modified parameter for retro simulation: $\tan \delta=7.7 \cdot 10^{-3}$ $g_{1}=g_{2}=1.95 \mathrm{~mm}(\mathrm{~b})$ Scatterer $\mathrm{n}^{\circ} 2, f_{0}=5.26 \mathrm{GHz}, \mathrm{AR}=0.73(-2.7 \mathrm{~dB})$. Modified parameter for retro simulation: $\tan \delta=8.1 \cdot 10^{-3}, L_{2}=16 \mathrm{~mm}$.
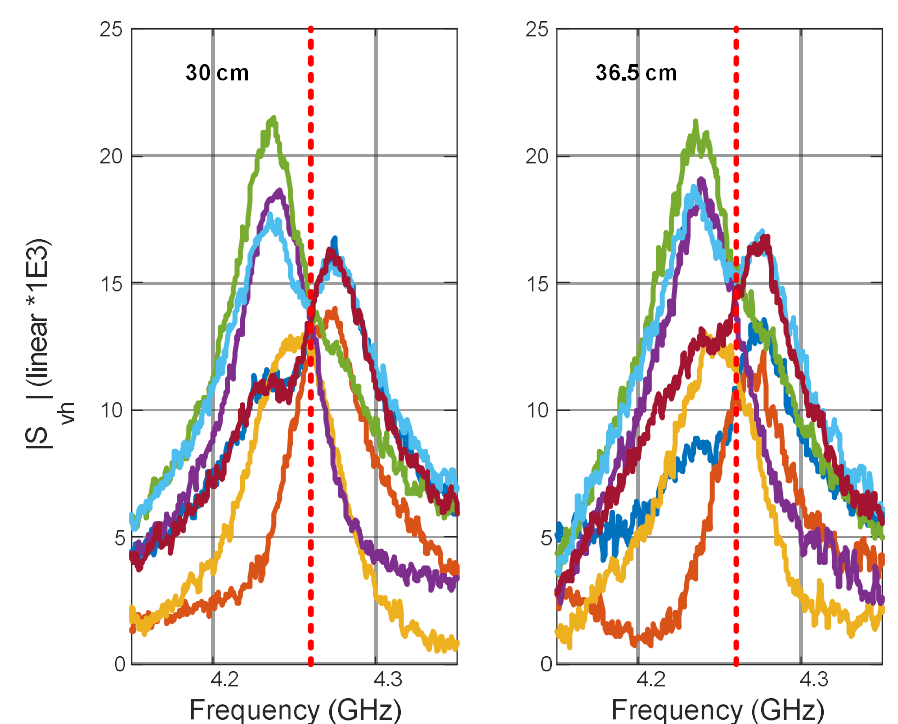

Fig. 13. Measurement of $S_{v h}$ for different angles and different distances between the scatterer and the antenna.
The value of $S_{v h}$ at the operating frequency $f_{0}$ is reported for different values of $\theta$ in Fig. 12, for the two scatterers. As expected from the theory, the trajectory of $S_{v h}(\theta)$ is describing a centered ellipse in both cases.

At nominal dimensions (table I), the quality factor of the measured resonators appears to be lower than expected in simulation, which results in a lower amplitude and a widening of the backscattered peak. A series of retro simulations has been undertaken to improve the agreement between simulation and measurement. The modified parameters obtained by fitting the measurement are indicated in the caption of Fig. 12. The ellipses obtained from this new set of parameters have been plotted in Fig. 10 and we observe a good agreement with the measurement. Note that due to the large number of independent geometric parameters, other sets of parameters could potentially lead to similar results.

\section{Effect of distance}

The impact of the distance $r$ between the scatterer $n^{\circ} 1$ and the antenna has been evaluated by measurements. The distance $r$ has been gradually increased from $30 \mathrm{~cm}$ to $49.5 \mathrm{~cm}$ and the measurement results are shown in Fig. 13 for different angles. The measurement results show that $S_{v h}$ which is normalized with respect to distance using (24) is almost constant. The comparison between the peak apex magnitude of the $60^{\circ}$ curve (in green in Fig. 13) gives a maximum relative error inferior to $6 \%$ for $42 \mathrm{~cm}$ and $49.5 \mathrm{~cm}$.

The total received power is composed of the power backscattered by the scatterer, which depends on the distance, as well as an additive white noise of constant power. Due to normalization (24), the attenuation of the signal to noise ratio (SNR) with respect to distance can be visualized on the measurement results in Fig. 13. The variance of the residual noise has been estimated to be $0.021,0.044,0.084$ and 0.146 for $30 \mathrm{~cm}, 36.5 \mathrm{~cm} 43 \mathrm{~cm}$ and $59.5 \mathrm{~cm}$ respectively. The variance is thus almost proportional to $R^{4}$ as expected from the radar equation. The decrease in SNR results in a progressive degradation of the axial ratio from $0.79(-2 \mathrm{~dB})$ at $30 \mathrm{~cm}$ to 0.70 $(-3 \mathrm{~dB})$ at $50 \mathrm{~cm}$ for the scatterer $\mathrm{n}^{\circ} 1$. Note that for the measurements results represented in Fig. 13, the tag is located in the reactive near field of the antenna for $r=30 \mathrm{~cm}$ and in
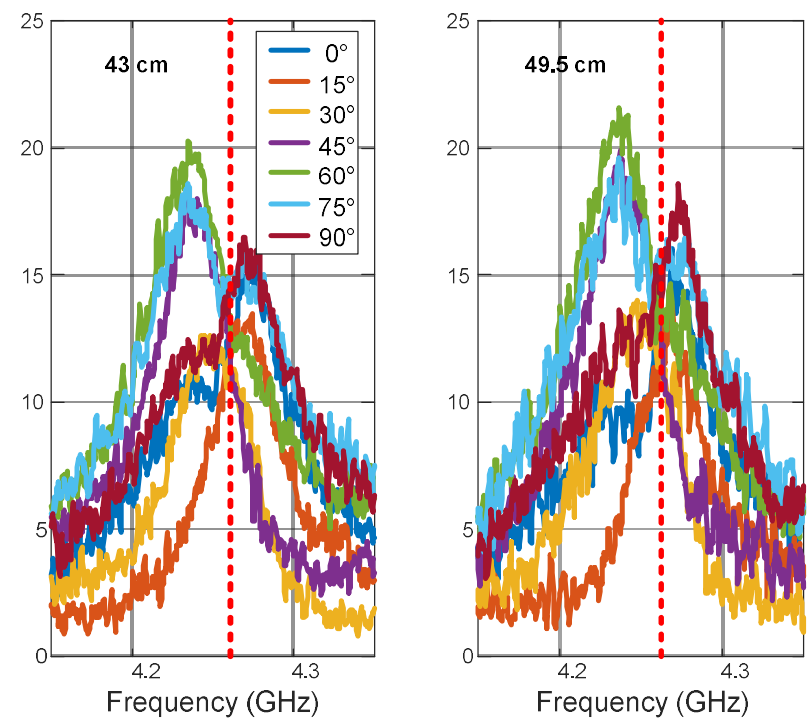
the far field of the antenna for $36.5 \mathrm{~cm} 43 \mathrm{~cm}$ and $49.5 \mathrm{~cm}$ without any significant difference between the measurements.

\section{CONCLUSION}

In the article, the analytic condition for which the $S_{v h}$ component of a general planar scatterer can be roll-invariant is first specified. Then, a simple method is proposed to design roll-invariant resonant scatterers from two microstrip dipoles slightly shifted in frequency and with a relative orientation of $45^{\circ}$. Two scatterers operating at $4.26 \mathrm{GHz}$ and $5.26 \mathrm{GHz}$ have been manufactured and measured providing good performances with an axial ratio inferior to $-2 \mathrm{~dB}$ and $-2.6 \mathrm{~dB}$ respectively. Although simple and illustrative, the design presented is sensitive to fabrication tolerance.

The scattering matrix obtained from (12) is compared with other targets producing cross-polarization components in the backscattered field in table II. The examination of the different scattering matrices shows that the roll invariant cross polarization (current work) is a feature that differs significantly from existing devices, such as polarization rotators or polarization converters, and which has not been addressed to date. The only other roll invariant target is the right or left Helix [15] which is a $3 \mathrm{D}$ target unlike the current work which is planar.

TABLE II

COMPARISON OF THE CURRENT WORK WITH OTHER CROSS POLARIZATION TARGETS

\begin{tabular}{|c|c|c|c|c|}
\hline Ref. & Description & $\begin{array}{l}\text { Implement } \\
\text { ation }\end{array}$ & $\begin{array}{c}\text { Roll } \\
\text { invariant }\end{array}$ & $\begin{array}{c}\text { Scattering } \\
\text { matrix S }\end{array}$ \\
\hline$[6]$ & $\begin{array}{l}\text { Reflection type } 90^{\circ} \\
\text { polarization rotator }\end{array}$ & $\begin{array}{c}\text {-FSS } \\
\text {-planar, } \\
\text { single layer }\end{array}$ & no & $\begin{array}{l}S\left(\theta=0^{\circ}\right) \\
=a\left[\begin{array}{ll}0 & 1 \\
1 & 0\end{array}\right]\end{array}$ \\
\hline [7] & $\begin{array}{l}\text { Reflection type } \\
\text { Linear to circular } \\
\text { polarization } \\
\text { converter }\end{array}$ & $\begin{array}{c}\text {-FSS } \\
\text {-planar, } \\
\text { Single layer }\end{array}$ & no & $\begin{array}{l}S\left(\theta=0^{\circ}\right) \\
=a\left[\begin{array}{cc}1 & \pm j \\
\pm j & b\end{array}\right]\end{array}$ \\
\hline [10] & $\begin{array}{l}\text { Depolarizing } \\
\text { chipless tag } \\
\text { (coupled dipoles) }\end{array}$ & $\begin{array}{l}\text {-planar, } \\
\text { single layer } \\
\text {-resonant }\end{array}$ & no & $\begin{array}{l}\mathrm{S}\left(\theta=45^{\circ}\right) \\
=\mathrm{a}\left[\begin{array}{ll}1 & 1 \\
1 & 1\end{array}\right]\end{array}$ \\
\hline [11] & $\begin{array}{l}\text { Wire mesh at } 45^{\circ} \\
\text { (calibration object) }\end{array}$ & $\begin{array}{c}\text {-FSS } \\
\text {-planar, } \\
\text { single layer }\end{array}$ & no & $\begin{array}{l}S\left(\theta=45^{\circ}\right) \\
=a\left[\begin{array}{ll}1 & 1 \\
1 & 1\end{array}\right]\end{array}$ \\
\hline [11] & $\begin{array}{c}\text { Dihedral corner at } \\
22.5^{\circ} \text { (calibration } \\
\text { object) }\end{array}$ & $-3 D$ & no & $\begin{array}{l}S\left(\theta=22.5^{\circ}\right) \\
=a\left[\begin{array}{cc}-1 & 1 \\
1 & 1\end{array}\right]\end{array}$ \\
\hline [15] & $\begin{array}{c}\text { Right or left Helix } \\
\text { antenna used as } \\
\text { scatterer. }\end{array}$ & $-3 D$ & yes & $\begin{array}{l}S \\
=a\left[\begin{array}{cc}1 & \pm j \\
\pm j & -1\end{array}\right]\end{array}$ \\
\hline & This work & $\begin{array}{l}\text {-planar, } \\
\text { single layer } \\
\text {-resonant }\end{array}$ & yes & $\begin{array}{l}S \\
=a\left[\begin{array}{ll}1 & 0 \\
0 & 1\end{array}\right] \\
+b\left[\begin{array}{cc}1 & \pm j \\
\pm j & -1\end{array}\right]\end{array}$ \\
\hline
\end{tabular}

\section{REFERENCES}

[1] C. R. Smith and P. M. Goggans, 'Radar target identification', IEEE Antennas Propag. Mag., vol. 35, no. 2, pp. 27-38, Apr. 1993.

[2] S.-W. Chen, Y.-Z. Li, X.-S. Wang, S.-P. Xiao, and M. Sato, 'Modeling and Interpretation of Scattering Mechanisms in Polarimetric Synthetic Aperture Radar: Advances and perspectives', IEEE Signal Process. Mag., vol. 31, no. 4, pp. 79-89, Jul. 2014.
[3] S. R. Cloude and E. Pottier, 'An entropy based classification scheme for land applications of polarimetric SAR', IEEE Trans. Geosci. Remote Sens., vol. 35, no. 1, pp. 68-78, Jan. 1997.

[4] R. Touzi, 'Target Scattering Decomposition in Terms of Roll-Invariant Target Parameters', IEEE Trans. Geosci. Remote Sens., vol. 45, no. 1, pp. 73-84, Jan. 2007.

[5] R. Paladini, L. Ferro Famil, E. Pottier, M. Martorella, F. Berizzi, and E. Dalle Mese, 'Point Target Classification via Fast Lossless and Sufficient

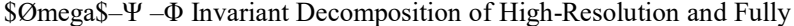
Polarimetric SAR/ISAR Data', Proc. IEEE, vol. 101, no. 3, pp. 798-830, Mar. 2013.

[6] X. Gao, X. Han, W.-P. Cao, H. O. Li, H. F. Ma, and T. J. Cui, 'Ultrawideband and High-Efficiency Linear Polarization Converter Based on Double V-Shaped Metasurface', IEEE Trans. Antennas Propag., vol. 63, no. 8, pp. 3522-3530, Aug. 2015.

[7] E. Doumanis, G. Goussetis, J. L. Gomez-Tornero, R. Cahill, and V. Fusco, 'Anisotropic Impedance Surfaces for Linear to Circular Polarization Conversion', IEEE Trans. Antennas Propag., vol. 60, no. 1, pp. 212-219, Jan. 2012.

[8] F. Samadi, M. Akbari, M. R. Chaharmir, and A. Sebak, 'Scatterer Surface Design for Wave Scattering Application', IEEE Trans. Antennas Propag., vol. 67, no. 2, pp. 1202-1211, Feb. 2019.

[9] A. Edalati and K. Sarabandi, 'Wideband, Wide Angle, Polarization Independent RCS Reduction Using Nonabsorptive Miniaturized-Element Frequency Selective Surfaces', IEEE Trans. Antennas Propag., vol. 62, no. 2, pp. 747-754, Feb. 2014.

[10] A. Vena, E. Perret, and S. Tedjni, 'A Depolarizing Chipless RFID Tag for Robust Detection and Its FCC Compliant UWB Reading System', IEEE Trans. Microw. Theory Tech., vol. 61, no. 8, pp. 2982-2994, Aug. 2013.

[11] C. J. Bradley et al., 'An investigation of bistatic calibration objects', IEEE Trans. Geosci. Remote Sens., vol. 43, no. 10, pp. 2177-2184, Oct. 2005.

[12] K. Sarabandi, F. T. Ulaby, and M. A. Tassoudji, 'Calibration of polarimetric radar systems with good polarization isolation', IEEE Trans. Geosci. Remote Sens., vol. 28, no. 1, pp. 70-75, Jan. 1990.

[13] W. W. Salisbury, 'Absorbent body for electromagnetic waves', US2599944A, Jun. 10, 1952.

[14] A. Mackay, 'Proof of polarisation independence and nonexistence of crosspolar terms for targets presenting $n$-fold $(n>2)$ rotational symmetry with special reference to frequency-selective surfaces', Electron. Lett., vol. 25 , no. 24 , pp. 1624-1625, Nov. 1989.

[15] E. Krogager, 'New decomposition of the radar target scattering matrix', Electron. Lett., vol. 26, no. 18, pp. 1525-1527, Aug. 1990.

[16] D. R. Jackson and N. G. Alexopoulos, 'Simple approximate formulas for input resistance, bandwidth, and efficiency of a resonant rectangular patch', IEEE Trans. Antennas Propag., vol. 39, no. 3, pp. 407-410, Mar. 1991.

[17] A. Yaghjian, 'An overview of near-field antenna measurements', IEEE Trans. Antennas Propag., vol. 34, no. 1, pp. 30-45, Jan. 1986.

[18] A. Ramos, E. Perret, O. Rance, S. Tedjini, A. Lázaro, and D. Girbau, 'Temporal Separation Detection for Chipless Depolarizing FrequencyCoded RFID', IEEE Trans. Microw. Theory Tech., vol. 64, no. 7, pp. 2326-2337, Jul. 2016. 